\title{
Modulus and Phase of the Reduced Logarithmic Derivative of the Cylindrical Bessel Function
}

\section{By Andrés Cruz and Javier Sesma}

\begin{abstract}
The modulus and phase of the reduced logarithmic derivative of the cylindrical Bessel function

$$
z J_{\nu}^{\prime}(z) / J_{\nu}(z)
$$

for complex variable $z$ and real order $\nu$, are investigated. Special attention is paid to the location of saddle points and their trajectories as $\nu$ varies.
\end{abstract}

1. Introduction. Quantum scattering by optical potentials is of considerable interest in that part of nuclear physics known as heavy-ion physics. The analysis of some mathematical aspects of quantum scattering, such as those discussed in a recent paper by the present authors [1], requires a good knowledge of the reduced logarithmic derivative of the cylindrical Bessel function

$$
F_{\nu}(z) \equiv z J_{\nu}^{\prime}(z) / J_{\nu}(z)
$$

for complex variable $z$ and positive half-integer index $\nu$ (physical angular momentum $l=\nu-1 / 2$ ). Speedy preliminary approximate solutions of the Schrödinger equation are greatly facilitated by a plot of the modulus and phase of $F_{\nu}(z)$ in the complex $z$ plane. In this paper we discuss the main features of such a plot for real values of the index $\nu$ going from $-\infty$ to $+\infty$.

The recurrence relation for the Bessel functions [2, Eq. 9.1.27] allows us to write

$$
F_{\nu}(z)=\nu-z J_{\nu+1}(z) / J_{\nu}(z)
$$

Since $J_{\nu}(z)$ is, for real $\nu$, a real function of $z$ [2, Eq.9.1.40], we have obviously

$$
F_{\nu}(\bar{z})=\overline{F_{\nu}(z)}, \quad \nu \text { real, }
$$

where the bar denotes complex conjugate. Furthermore, the analytic continuation of $J_{\nu}(z)$ [2, Eq. 9.1.35] gives

$$
F_{\nu}(-z)=F_{\nu}(z), \quad \nu \text { real }
$$

Received December 10, 1979.

AMS (MOS) subject classifications (1970). Primary 33A40; Secondary 65H05, 81 A45.

Key words and phrases. Cylindrical Bessel functions, modulus and phase of the reduced logarithmic derivative, quantum potential scattering. 
In view of (1.3) and (1.4), we need consider only the first quadrant in the $z$-plane, the two positive semiaxes included. Moreover, it is evident that $F_{\nu}(z)$ is real on these two semiaxes.

For negative integer values of the index $\nu$, the relation [2, Eq. 9.1.5]

$$
J_{-n}(z)=(-1)^{n} J_{n}(z), \quad n \text { integer, }
$$

substituted in Eq. (1.2), gives immediately

$$
F_{-n}(z)=-F_{n}(z), \quad n \text { an integer } \neq 0
$$

Knowledge of the location of zeros, poles, and saddle points is of great aid for drawing a modulus and phase plot of $F_{\nu}(z)$. Zeros and poles are briefly reviewed in Section 2. Saddle points deserve a more detailed analysis in Section 3. Finally, some plots are presented in Section 4. Because of the symmetries contained in Eqs. (1.3) and (1.4), we consider only values of $z$ such that

$$
\operatorname{Re} z \geqslant 0, \quad \operatorname{Im} z \geqslant 0,
$$

in all what follows, unless otherwise explicitly stated.

2. Zeros and Poles. All the contour lines in the $z$-plane corresponding to constant phase of $F_{\nu}(z)$ pass through the zeros and poles of $F_{\nu}(z)$. The location of these points for real $\nu$ can be immediately obtained, as they correspond to the zeros of $J_{\nu}^{\prime}(z)$ and $J_{\nu}(z)$, respectively. These have been extensively studied in the past and tables are available in the literature. References can be found in classical treatises on special functions ([2, pp. 370-372 and 388-389], [3, pp. 146-147]) and in the more recent one by Luke [4, pp. 403-412]. To summarize, let us discuss the qualitative behavior of zeros and poles of $F_{\nu}(z)$ as $\nu$ varies from $+\infty$ to $-\infty$ through real values.

For real positive $\nu, F_{\nu}(z)$ has an infinity of real zeros and poles, to the right of the point $z=\nu$. Zeros and poles alternate from left to right, starting with a zero. As $\nu$ decreases, they all move along the real axis towards the origin. The first zero enters the origin for $\nu=0$. As $\nu$ goes from 0 to -1 , it moves on the imaginary axis, returning to the origin for $\nu=-1$. Also the first pole and the second zero reach the origin for $v=-1$. In general, whenever $v$ approaches a negative integer, $-m<v, m=$ $1,2,3, \ldots, 2(m+1)$ zeros and $2 m$ poles in the whole $z$-plane tend to the origin. As $v$ decreases below $-m$, they leave the origin, making a trip in the complex $z$-plane and coming back to the origin for $\nu=-(m+1)$. Two new (symmetric) real zeros and poles enter the origin for this value of $\nu$.

3. Saddle Points. Let us now investigate the location of points $z_{n}$ in which the modulus of $F_{\nu}(z)$ presents a saddle. In these points, one has

$$
d F_{\nu}(z) / d z=0 \quad \text { for } z=z_{n} .
$$

On the other hand, $F_{\nu}(z)$ satisfies the differential equation

$$
z d F_{\nu}(z) / d z+\left(F_{\nu}(z)\right)^{2}+z^{2}-\nu^{2}=0,
$$


obtained immediately from the Bessel differential equation [2, Eq. 9.1.1] and the definition (1.1). The condition (3.1), substituted in (3.2), gives for the saddle points

$$
\left(F_{\nu}\left(z_{n}\right)\right)^{2}=\nu^{2}-z_{n}^{2}
$$

This relation can be used to obtain numerically the values $z_{n}$. For the calculation of $F_{\nu}(z),(1.2)$, use of a continued fraction expansion [2, Eq. 9.1.73] for the quotient of Bessel functions seems to be the most convenient. The convergence of such continued fraction expansions has been recently studied by Gautschi and Slavik [5]. Alternatively, $F_{\nu}(z)$ can be evaluated using rational approximations [4, pp. $364 \mathrm{ff}$.]. In this way, and using the "steepest descent" method to solve (3.3), we have obtained the lowest values of $z_{n}$ for varying $\nu$. The results are shown in Figures 1 and 2 .
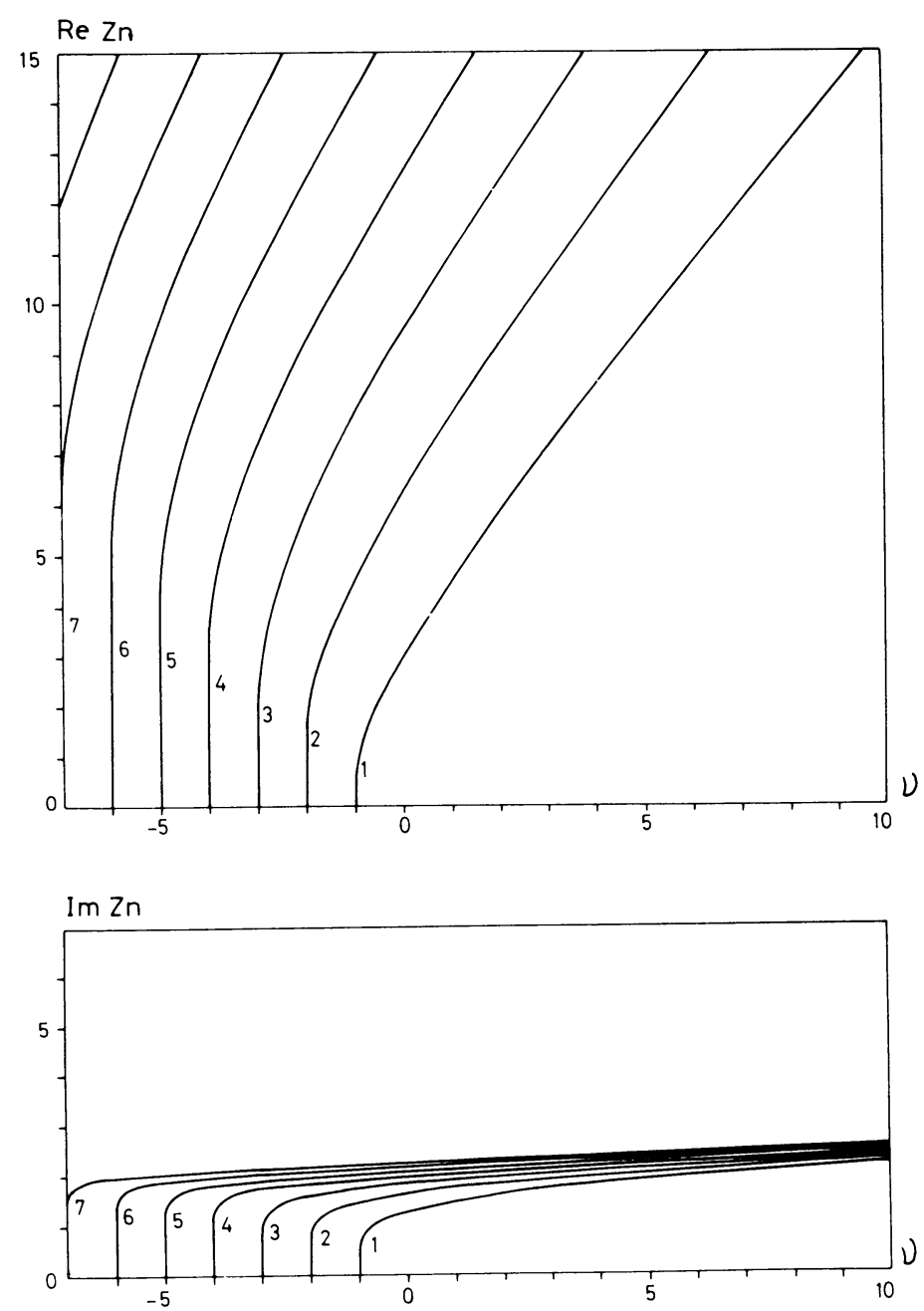

FIGURE 1

Saddle points, $z_{n}$, of the reduced logarithmic derivative of the cylindrical Bessel function. The real and imaginary parts of the first seven saddle points are drawn as functions of the order $\nu$. For $v<-n$, the location of the $n$th saddle point is shown in Figure 2. 
1320

ANDRES CRUZ AND JAVIER SESMA
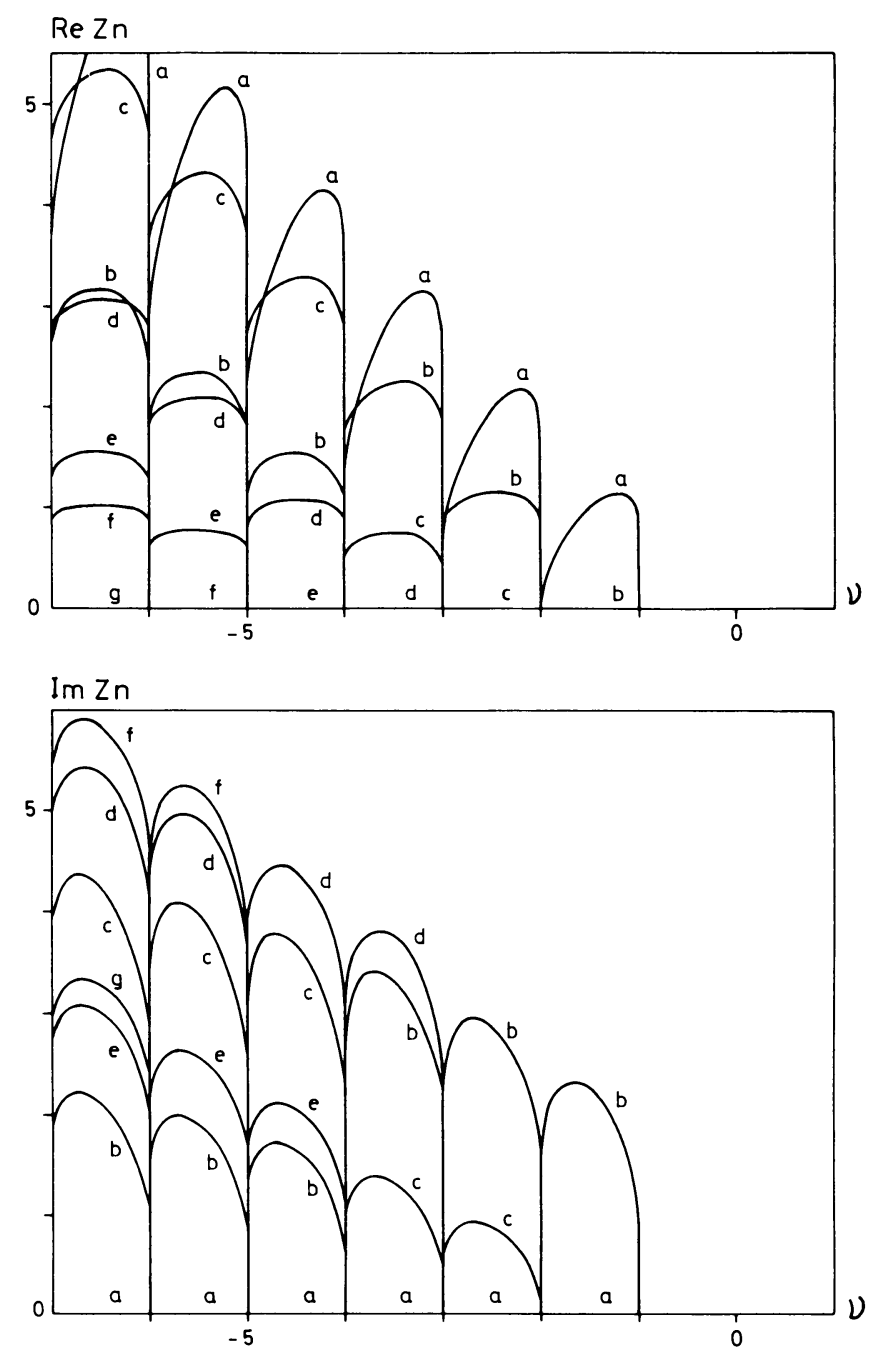

FIGURE 2

Saddle points, $z_{n}$, of the reduced logarithmic derivative of the cylindrical Bessel function. The behavior is shown of the real and imaginary parts of the saddle points as $\nu$ varies between two consecutive negative integers. This figure complements Figure 1.

It is not difficult to find analytic expressions giving the values $z_{n}$ to desirable accuracy. A possible procedure is that used by Fettis et al. [6] to obtain the saddle points of the complementary error function. The method consists, basically, of expanding $F_{\nu}(z)$ as a Taylor series in the vicinity of the saddle point and, after that, inverting the series. Using a more convenient notation

$$
s \equiv z^{2}, \quad G_{\nu}(s) \equiv F_{\nu}(z),
$$

one obtains for the Taylor expansion

$$
\begin{aligned}
G_{\nu}(s)= & g_{n}-\left(2 ! 2 s_{n}\right)^{-1}\left(s-s_{n}\right)^{2}+\left(3 ! 2 s_{n}^{2}\right)^{-1}\left(g_{n}+2\right)\left(s-s_{n}\right)^{3} \\
& -\left(4 ! 2 s^{3}\right)^{-1}\left(g_{n}+2\right)\left(g_{n}+3\right)\left(s-s_{n}\right)^{4}+\cdots,
\end{aligned}
$$


where we have denoted

$$
g_{n} \equiv\left(\nu^{2}-s_{n}\right)^{1 / 2} .
$$

By expanding $g_{n}$ and $s_{n}^{-m}$ in power series of $\left(s-s_{n}\right),(3.5)$ becomes

$$
\begin{aligned}
G_{\nu}(s)= & g+\left(s-s_{n}\right)(2 g)^{-1}-\left(s-s_{n}\right)^{2} 4^{-1}\left[\left(2 g^{3}\right)^{-1}+s^{-1}\right] \\
& +\left(s-s_{n}\right)^{3} 4^{-1}\left[\left(4 g^{5}\right)^{-1}+(g-1)\left(3 s^{2}\right)^{-1}\right] \\
& -\left(s-s_{n}\right)^{4} 4^{-1}\left\{5\left(32 g^{7}\right)^{-1}+[1+(2+g)(g-5) / 12] s^{-3}\right. \\
& \left.-\left(6 g s^{2}\right)^{-1}\right\}+\cdots,
\end{aligned}
$$

with the abbreviation

$$
g \equiv\left(v^{2}-s\right)^{1 / 2} .
$$

Let us now introduce a new variable,

$$
t \equiv G_{\nu}(s)-g,
$$

vanishing at the saddle points. The inversion of the series in (3.7) gives

$$
s_{n}=s-b_{1} t-b_{2} t^{2}-b_{3} t^{3}-b_{4} t^{4} \ldots,
$$

where

(3.11, a) $\quad b_{1}=2 g$,

$(3.11, \mathrm{~b}) \quad b_{2}=1+2 g^{3} / \mathrm{s}$,

$(3.11, \mathrm{c}) \quad b_{3}=\left(4 g^{2} / s\right)\left[1+g^{2}(1+2 g) / 3 s\right]$,

$(3.11, \mathrm{~d}) \quad b_{4}=(g / 3 s)\left\{15 / 2+\left(g^{2} / s\right)\left[10+31 g+\left(g^{2} / s\right)\left(4+14 g+12 g^{2}\right)\right]\right\}$,

The repeated application of (3.10), starting with a value $s$ not far from $s_{n}$, allows one to obtain $s_{n}$ with the desired accuracy.

The symmetry property given by (1.6) causes some saddle points to enter the origin whenever $v$ reaches negative integer values. Let us study in which form these saddle points tend to the origin as $\nu$ tends to $-m$.

If we replace (1.2) in (3.3), we obtain for the saddle points either of the two conditions

$$
\begin{aligned}
& z_{n} J_{\nu+1}\left(z_{n}\right) / J_{\nu}\left(z_{n}\right)=\nu+\left(\nu^{2}-z_{n}\right)^{1 / 2}, \\
& z_{n} J_{\nu+1}\left(z_{n}\right) / J_{\nu}\left(z_{n}\right)=\nu-\left(\nu^{2}-z_{n}^{2}\right)^{1 / 2} .
\end{aligned}
$$

For values of $\nu$ near negative integers,

$$
\nu=-m+\epsilon, \quad|\epsilon|<<1,
$$

we can use approximate expressions, deduced from the usual ascending series expansion of the Bessel functions, to obtain from $(3.12$, a)

$$
z_{n} \simeq 2[-m(m+1) ! m ! \epsilon]^{1 / 2(m+1)}, \quad m=1,2,3, \ldots,
$$

and from $(3.12, \mathrm{~b})$

$$
z_{n} \simeq 2[(m-1) !(m-2) ! \epsilon / m]^{1 / 2(m-1)}, \quad m=2,3,4, \ldots .
$$


From these two equations we see that, as $\nu$ tends to a negative integer value, $-m$, saddle points tend to the origin along the straight lines

$$
\operatorname{Arg} z=k \pi / 2(m+1), \quad k=1,3,5, \ldots \leqslant m+1,
$$

$$
\operatorname{Arg} z=k \pi / 2(m-1), \quad k=0,2,4, \ldots \leqslant m-1, m>1,
$$

for $\epsilon>0$, and along

$$
\operatorname{Arg} z=k \pi / 2(m+1), \quad k=0,2,4, \ldots \leqslant m+1,
$$

$$
\operatorname{Arg} z=k \pi / 2(m-1), \quad k=1,3,5, \ldots \leqslant m-1,
$$

for $\epsilon<0$. The trajectories of these saddle points leaving the origin as $v$ decreases from $-m$ and coming back to the origin as $\nu$ reaches the value $-m-1$ are shown in Figure 2. It is interesting to notice that, for $\nu<-1$, there is one saddle point on the positive real semiaxis and another one on the positive imaginary semiaxis.

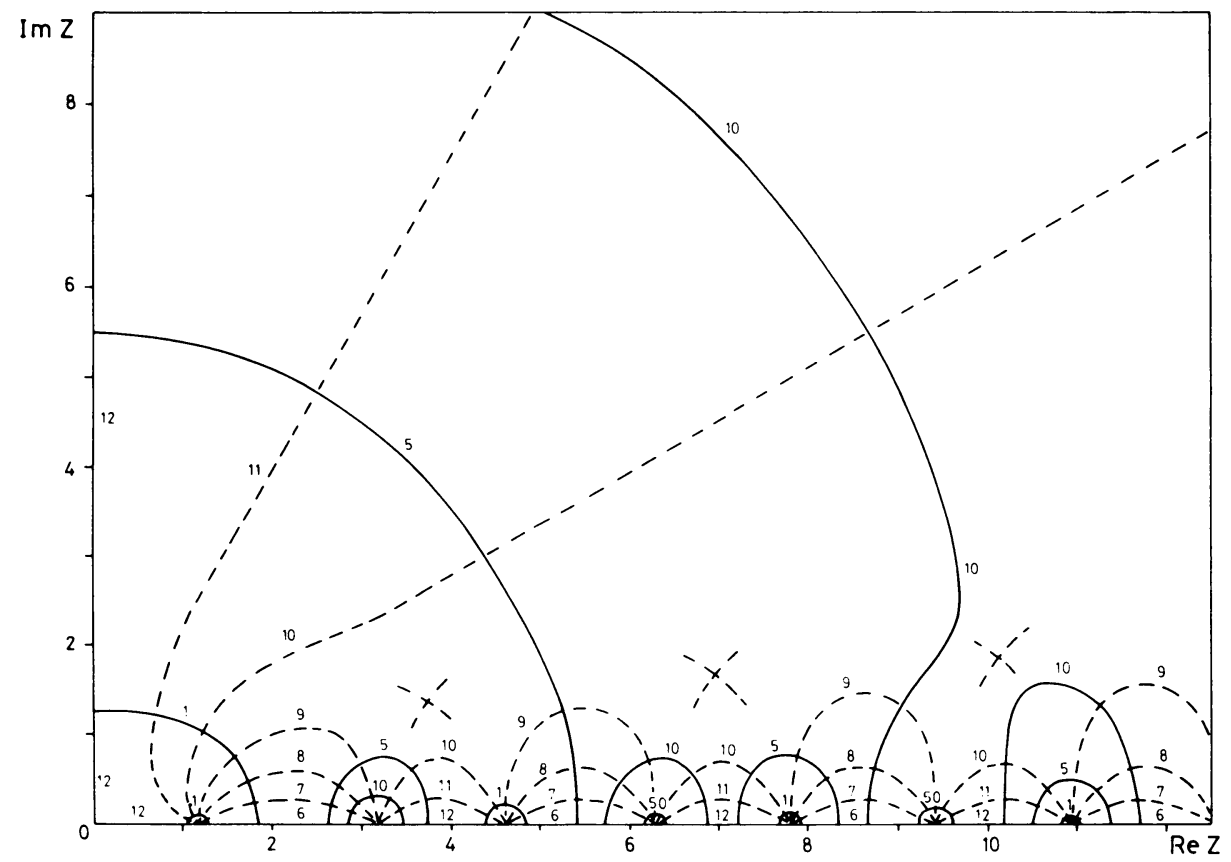

FIGURE 3

Modulus and phase of the reduced logarithmic derivative of the cylindrical Bessel function of order $\nu=.5$. The numbers aside lines indicate the value of the modulus (continuous lines) or the phase in units $\pi / 6$ (dashed lines). The constant phase lines connect zeros and poles. Saddle points are indicated by the intersection of their constant phase lines.

4. Modulus and Phase Plots. As an illustration, we present, in Figures 3 and 4, plots of the modulus and phase of $F_{\nu}(z)$ for $\nu=.5$ and $\nu=-5.5$, respectively. The contour lines corresponding to constant modulus or constant phase have been obtained numerically from (1.2), with a continued fraction expansion for the quotient of the Bessel functions. Of course, both families of modulus and phase lines are orthogonal. In the 
figures we have marked the position of the saddle points. The modulus and phase of $F_{\nu}(z)$ at these points come immediately from (3.3).

The modification required by the plots as $v$ varies can be easily deduced from the discussion concerning zeros, poles, and saddle points done in Sections 2 and 3.

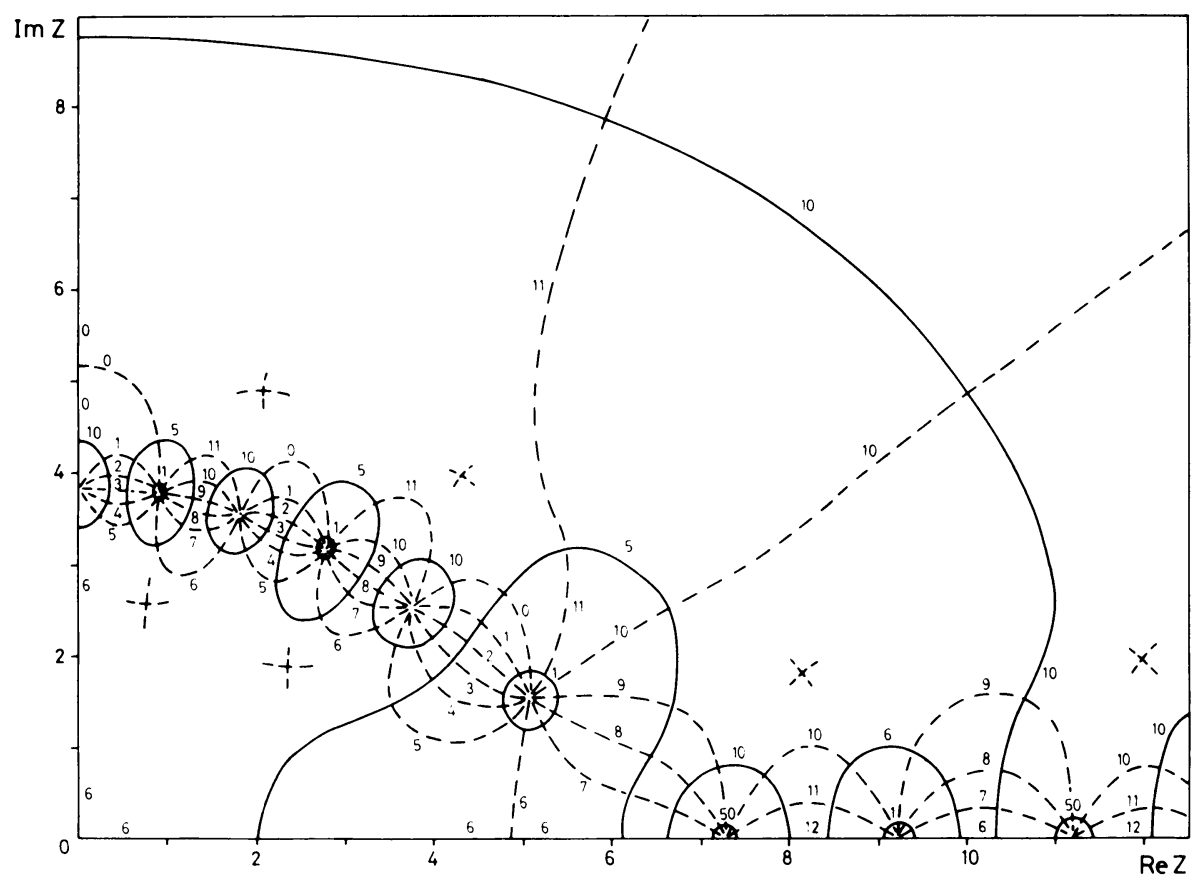

\section{FigURE 4}

Modulus and phase of the reduced logarithmic derivative of the cylindrical Bessel function of order $v=-5.5$. The notation is the same as in Figure 3.

Acknowledgments. This research has been sponsored by Instituto de Estudios Nucleares. One of the authors (J.S.) is very indebted to Professor P. E. Hodgson for hospitality at the Oxford Nuclear Physics Laboratory and to Dr. F. Brieva for decisive help in the computational task. The other author thanks Professor R.J.N. Phillips for the hospitality of the Theory Division of the Rutherford Laboratory.

Departamento de Física Teórica and

Instituto de Física Nuclear y Altas Energías

Facultad de Ciencias

Universidad de Zaragoza

Zaragoza, Spain

Nuclear Physics Laboratory

University of Oxford

Oxford, United Kingdom

1. A . CRUZ \& J. SESMA, "Convergence of the Debye expansion for the $S$ matrix," $J$. Math. Phys., v. 20, 1979, pp. 126-131.

2. M. ABRAMOWITZ \& I. A. STEGUN (Editors), Handbook of Mathematical Functions, Dover, New York, 1965. 
3. W. MAGNUS, F. OBERHETTINGER \& R. P. SONI, Formulas and Theorems for the Special Functions of Mathematical Physics, Springer-Verlag, New York, 1966.

4. Y. L. LUKE, Mathematical Functions and their Approximations, Academic Press, New York, 1975.

5. W. GAUTSCHI \& J. SLAVIK, "On the computation of modified Bessel function ratios," Math. Comp., v. 32, 1978, pp. 865-875.

6. H. E. FETTIS, J. C. CASLIN \& K. R. CRAMER, "Saddle points of the complementary error function," Math. Comp., v. 27, 1973, pp. 409-412. 